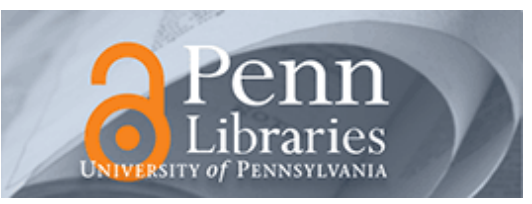

University of Pennsylvania

ScholarlyCommons

$4-11-2011$

\title{
Motor Sizing for Legged Robots Using Dynamic Task Specification
}

\author{
Avik De \\ University of Pennsylvania \\ Goran Lynch \\ University of Pennsylvania \\ Aaron Johnson \\ University of Pennsylvania \\ Daniel E. Koditschek \\ University of Pennsylvania, kod@seas.upenn.edu
}

Follow this and additional works at: https://repository.upenn.edu/ese_papers

Part of the Other Electrical and Computer Engineering Commons

\section{Recommended Citation}

Avik De, Goran Lynch, Aaron Johnson, and Daniel E. Koditschek, "Motor Sizing for Legged Robots Using Dynamic Task Specification", . April 2011.

De, A., Lynch, G., Johnson, A., Koditschek, D. (2011). "Motor sizing for legged robots using dynamic task specification," IEEE Conference on Technologies for Practical Robot Applications (TePRA), 2011, pp.64-69, 11-12 April 2011. doi: 10.1109/TEPRA.2011.5753483

(c) 2011 IEEE. Personal use of this material is permitted. Permission from IEEE must be obtained for all other uses, in any current or future media, including reprinting/republishing this material for advertising or promotional purposes, creating new collective works, for resale or redistribution to servers or lists, or reuse of any copyrighted component of this work in other works.

This paper is posted at ScholarlyCommons. https://repository.upenn.edu/ese_papers/611

For more information, please contact repository@pobox.upenn.edu. 


\title{
Motor Sizing for Legged Robots Using Dynamic Task Specification
}

\begin{abstract}
We explore an approach to incorporating task and motor thermal dynamics in the selection of actuators for legged robots, using both analytical and simulation methods. We develop a motor model with a thermal component and apply it to a vertical climbing task; in the process, we optimally choose gear ratio and therefore eliminate it as a design parameter. This approach permits an analytical proof that continuous operation yields superior thermal performance to intermittent operation. We compare the results of motor sizing using our proposed method with more conventional techniques such as using the continuously permissible current specification. Our simulations are run across a database of commercially available motors, and we envision that our results might be of immediate use to robot designers for motor as well as gearbox selection.
\end{abstract}

\section{Keywords}

motor sizing, locomotion, intermittent, continuous, thermal

Disciplines

Electrical and Computer Engineering | Other Electrical and Computer Engineering

\section{Comments}

De, A., Lynch, G., Johnson, A., Koditschek, D. (2011). "Motor sizing for legged robots using dynamic task specification," IEEE Conference on Technologies for Practical Robot Applications (TePRA), 2011, pp.64-69, 11-12 April 2011. doi: 10.1109/TEPRA.2011.5753483

(C) 2011 IEEE. Personal use of this material is permitted. Permission from IEEE must be obtained for all other uses, in any current or future media, including reprinting/republishing this material for advertising or promotional purposes, creating new collective works, for resale or redistribution to servers or lists, or reuse of any copyrighted component of this work in other works. 


\title{
Motor Sizing for Legged Robots Using Dynamic Task Specification
}

\author{
Avik De*, Goran Lynch*, Aaron Johnson*, Daniel Koditschek* \\ * Electrical and Systems Engineering Department, University of Pennsylvania \\ 200 S. 33rd St, Moore 203, Philadelphia, PA, 19104. Phone: (215)898-9241 \\ \{avik,goran,aaronjoh,kod\}@ seas.upenn.edu
}

\begin{abstract}
We explore an approach to incorporating task and motor thermal dynamics in the selection of actuators for legged robots, using both analytical and simulation methods. We develop a motor model with a thermal component and apply it to a vertical climbing task; in the process, we optimally choose gear ratio and therefore eliminate it as a design parameter. This approach permits an analytical proof that continuous operation yields superior thermal performance to intermittent operation. We compare the results of motor sizing using our proposed method with more conventional techniques such as using the continuously permissible current specification. Our simulations are run across a database of commercially available motors, and we envision that our results might be of immediate use to robot designers for motor as well as gearbox selection.
\end{abstract}

\section{INTRODUCTION}

Choosing the appropriate motors for an autonomous legged robot poses challenges not typically faced by designers in other domains of mechatronics and electromechanical automation. The highly varied and fundamentally intermittent nature of a running, climbing, or crawling machine's interactions with its environment combined with the severe limitations of contemporary actuator power density present a narrowly constrained set of design choices, whose constraints are at the same time very hard to characterize. Currently, many designers rely heavily on empirical trial and error; many versions of a robot are built with iteratively better motor/gearbox choices. ${ }^{1}$ Another method is to pick a single speed/torque operating point and find a motor that can achieve this at continuous steady-state operation [3]. Other, more considered approaches to motor sizing employ dynamical simulations of the robot in question [4], [5], [6], whereby a significant component of the empirical "generate and test" cycle can be iterated in software. In this work we are motivated to develop a still-more rational approach to motor sizing, attempting to develop mathematically generated (hence, a priori formally guaranteed) guidelines while enforcing constraints that arise from the interaction of motor dynamics and thermal behavior with the task assigned to the robot.

Rational selection of robot motors and gearboxes has traditionally been driven by industrial applications, in which a

\footnotetext{
${ }^{1}$ This design procedure was required for all the usefully working machines whose development we are familiar with-EduBot, RiSE, DynoClimber [1], and most recently X-RHex [2]-and we strongly suspect, most of the legged robots that have demonstrated capable locomotion in unstructured, outdoor environments
}

task typically consists of a known target trajectory [7] (eg. a robotic arm in an assembly line lifts and precisely maneuvers a specified part) accomplished by an amply-sized actuator. However, in the realm of mobile, legged robots, motor capabilities (and damage/failure conditions) and robot behavior (leg trajectories) can not be so neatly divorced. Hence, we address two coupled problems: the representation of a motor operating regime, and a model of the thermal consequences of that operational mode.

The motor selection methodology explored here is motivated by the experience of iterative motor selection for $\mathrm{X}$ RHex [2] and operating-point-based selection for its predecessors. Specifically, coupling the electromechanical to the thermal models, we outline a design methodology that selects the best performance from our actuator while avoiding thermal damage (including selection of the optimal gear ratio). We run numerical simulations over the space of commercially available motors and demonstrate how our algorithm produces results notably different from conventional motor sizing methods that typically either ignore thermal considerations entirely or impose overly conservative current limits based upon the permissible ceiling associated with continuous steady state operation.

\section{Problem Statement}

We apply our thermal-mechanical coupled motor models to a climbing problem by positing a scenario in which the actuator must lift a constant mass vertically against gravity, absent any friction.

We characterize the operating regime by substituting for the traditional library of typical task trajectories a single dynamical model that generates-at least in caricature-the entire family of speed-torque challenges likely to be encountered over the task domain. In this preliminary work we commit to the very specific task domain described above, and limit the design choices to varied control policies in the following manner: the motor can either be running continuously, in which mode a constant voltage is applied to the motor terminals, or intermittently, wherein the motor switches between operation at a constant voltage and being disconnected from the system (applies no force). The continuous operation mode can be thought of as representing a robot with wheels rolling up a pole, while the intermittent operation mode corresponds to a legged robot that bounds or leaps upward. Within this 
very specific task domain, we formally characterize the manner in which legged robots (morphologically constrained to intermittent loading) pose a fundamentally different set of requirements for motor selection than do wheeled robots (in which continuous power delivery is acceptable).

We use a motor thermal model [3], [2], [8] to characterize the thermal penalties of task achievement. By seeking optimal performance while satisfying thermal constraints, we can evaluate motors based both on their ability to accomplish a given task while incorporating some view of the performance quality they afford.

\section{AnAlyticAl RESUlts}

\section{A. Modeling}

1) Motor Reparameterization: The salient characteristics of a particular motor used in a robot are heavily dependent on the gearbox placed at its output. In this work we want to focus on the intrinsic properties of a motor which affect its performance across operational regimes, so our approach will be to normalize the effect by choosing the best possible gearbox for the motor to perform the task. The author of [7] investigated the gearbox-independent capabilities of motors for a known output trajectory; we address a similar question for a specific motor and a known dynamical ("task") model.

A typical motor operating at its nominal voltage operates along a speed-torque curve determined fully by the stall torque $\tau_{s}$ and no-load speed $\omega_{\mathrm{nl}}$ [9], [5] . Whereas the corresponding stall torque and no load speed after gearing are both affected by the reduction ratio $G$, the peak mechanical power,

$$
P=\frac{\tau_{s} \omega_{\mathrm{nl}}}{4}
$$

is not.

We now assume that the angular displacement of the output shaft is proportional to vertical displacement of the mass. This is equivalent to the introduction of a rack-and-pinion transmission with selected pinion radius $R_{p}$. Our actuator, consisting of the motor and two-stage transmission, has a noload speed and stall force given by

$$
v_{\mathrm{nl}}=\frac{R_{p} \omega_{\mathrm{nl}}}{G} \quad \text { and } \quad F_{s}=\frac{4 P}{v_{\mathrm{nl}}}
$$

These parameters define an output speed-force curve, along which the actuator operates.

We will choose $\left(P, v_{\mathrm{nl}}\right)$ as our motor operating parameterization. Note that the effect of varying the reduction ratio is completely captured in the scalar parameter $v_{\mathrm{nl}}$, and we optimize over all possible values it can take in Section III-C.

2) Actuator Dynamics: Our task involves lifting a mass $m$ (includes motor and payload) vertically. If $v$ is the vertical speed of the mass $m$, the relationship between vertical speed and upward force on the mass can be written as

$$
\frac{v}{v_{\mathrm{nl}}}=1-\frac{F}{F_{s}},
$$

since any achievable pair $(F, v)$ lies on the output speed-force line with intercepts at $\left(F_{s}, 0\right)$ and $\left(0, v_{\mathrm{nl}}\right)$.
Accounting for gravity, the system dynamics are given by $F=m(g+\dot{v})$. Specifically, for the initial condition $v(0)=0$, we abbreviate the dependence upon the motor parameters by defining $a_{1}\left(v_{\mathrm{nl}}\right)=\frac{4 P}{m v_{\mathrm{nl}}}-g$ and $a_{2}\left(v_{\mathrm{nl}}\right)=\frac{4 P}{m v_{\mathrm{n}}^{2}}$. Using these substitutions, the system's explicit solution can be written concisely:

$$
v\left(t, v_{\mathrm{nl}}\right)=\frac{a_{1}}{a_{2}}\left(1-e^{-a_{2} t}\right),
$$

Taking the time derivative, $\dot{v}\left(t, v_{\mathrm{nl}}\right)=a_{1} e^{-a_{2} t}$.

3) Thermal Model: For thermal behavior, we use a second order dissipative thermal model excited by the coil current [3], [10], [2]. We are interested in the "thermal current" (which is the heat source as described in [2]),

$$
\iota\left(t, v_{\mathrm{nl}}\right)=i_{\mathrm{m}}^{2} R_{\text {coil }}=\frac{R_{\mathrm{coil}} v_{\mathrm{nl}}^{2} F\left(t, v_{\mathrm{nl}}\right)^{2}}{K_{T}^{2} \omega_{\mathrm{nl}}^{2}},
$$

where $K_{T}$ is the torque constant of the motor, $i_{\mathrm{m}}(t)$ is the coil current, and $F\left(t, v_{\mathrm{nl}}\right)$ is the force applied to the mass. Note that $R_{p}$, as defined above, cancels out and does not appear in this equation.

a) Full Thermal Model: The full thermal model can be written in state space as

$$
\dot{\rho}(t)=A \rho(t)+B \iota(t)+B_{0},
$$

where $\rho=\left(\rho_{\text {core }}, \rho_{\text {case }}\right)^{T}$ are the core and case temperatures,

$$
\begin{aligned}
& A=\left[\begin{array}{cc}
-\frac{1}{R_{1} C_{1}} & \frac{1}{R_{1} C_{1}} \\
\frac{1}{R_{1} C_{2}} & -\frac{1}{C_{2}}\left(\frac{1}{R_{1}}+\frac{1}{R_{2}}\right)
\end{array}\right], \\
& B=\left[\begin{array}{c}
\frac{1}{C_{1}} \\
0
\end{array}\right], \quad \text { and } \quad B_{0}=\left[\begin{array}{c}
0 \\
\frac{\rho_{\text {ambient }}}{R_{2} C_{2}}
\end{array}\right],
\end{aligned}
$$

where $R_{1} C_{1}$ and $R_{2} C_{2}$ are the motor's specified thermal time constants for core and case, respectively.

Considered as linear time invariant control system with input $\iota$ and output $\rho$, system (6) admits exact closed form solutions involving a convolution integral that depends upon the specific thermal input profile, $\iota(t)$. We next provide a simplification which allows a qualitative understanding of system behavior without explicit evaluation of this convolution integral for each specific thermal profile $\iota(t)$.

b) RMS Method: For typical motors, (6) acts as a low pass filter, attenuating frequencies greater than that specified by the lower system time constant (in this case, the smaller of the two real eigenvalues of $A$ that evaluates to approximately $10^{-2} \mathrm{rad} / \mathrm{s}$ for the typical range of motors found in the Maxon catalog [9]). For an intermittent input which is periodic in nature the thermal excitation can be re-expressed as a Fourier series, respecting which an aggressive simplification (motivated by this characterization of the thermal plant as a low-pass filter) suggests an approximation based solely upon the DC term,

$$
\bar{\iota}=\frac{R_{\text {coil }} \int_{0}^{T}\left(i_{\mathrm{m}}(t)\right)^{2} d t}{T},
$$

where $T$ is the time period. Note that this is the square of the RMS current, scaled by a constant. 
Under these assumptions the equation (6) has a constant input, and we can calculate steady state values by setting $\dot{\rho}=0$ to obtain the steady-state temperature

$$
\Delta \bar{\rho}_{\text {core }}\left(v_{\text {nl }}\right)=\left(R_{1}+R_{2}\right) \bar{\iota},
$$

where the bar denotes that it is the RMS solution, and the $\Delta$ denotes that it is the difference between the core and ambient temperatures.

\section{B. Intermittent Operation Incurs a Thermal Penalty}

We consider, analytically, the simple, constrained scenario arising from expending a fixed amount of mechanical energy in the shortest possible amount of time. We will prove that a motor operating at a constant velocity introduces less (wasteful) thermal energy than does a motor operating intermittently (with such operations' requisite variation in velocity). Furthermore, this result is true irrespective of the gearbox used with the intermittent motor, as long as the reduction ratio is chosen optimally in the continuous case.

Smoothing actuator power output is intuitively beneficial: thermal energy emitted by the motor coil is proportional to the square of motor torque, while at a given speed, motor power output is linearly dependent on output torque. Intermittent operation requires a motor to operate at slower speeds and higher torques for part of each stride; these higher torques incur a large thermal cost which, as we demonstrate, can not be outweighed by a refractory period.

On the other hand, we approach intermittent operation here with no assumption other than that the motor is disconnected from the mass at some point during task execution. It is not immediately obvious that every possible intermittent control strategy of engagement/disengagement will be thermally inferior to continuous steady-state operation, but we show that this is indeed the case.

We assume that, as is the case for a legged robot, intermittent operation is constrained morphologically (eg. ground contact occurs only periodically), yet, as would be the typical case for a well designed control policy, ${ }^{2}$ within any given "on" period, we wish to maximize output power and therefore apply constant (maximal) voltage to the motor terminals.

1) Comparing Cost of Mechanical Work: Whereas in Section III-C we compare the performance (vertical climbing speed) of different motors, in this section (for analytical simplicity) we are comparing the thermal cost of equal mechanical work in continuous and intermittent modes of operation for the same motor. We will formally show that intermittent operation must result in a higher thermal cost in this setting. As a corollary, if we lift the "equal work" restriction and instead restrict both continuous and intermittent systems to the same peak temperature, the intermittent system must, necessarily, do less work.

\footnotetext{
2 We are not presuming that all actuation effort is aimed at producing the maximal positive work. For example, animal locomotion typically does not support this presumption since muscles are as often used as "brakes" or "struts" as they are as "motors" [11]. We merely presuppose that effort to either oppose or advance the direction of mass motion, once recruited, will typically be as great as possible for its entire duration.
}

We denote the force exerted on the mass as $F_{c}(t)$ and its velocity as $v_{c}(t)$ for the continuous case, and $\left(F_{i}(t), v_{i}(t)\right)$ denote the analogous quantities in intermittent operation.

We assume that $F_{c}(t)>0$ for $t \in T$, and the range of $T$ is the maximum time interval of the experiment. We also assume that $F_{i}(t)>0$ for $t \in A$ ("active" time interval), where $A \subset T$ strictly. This means that for the time in $T \backslash A$ the motor is switched off (hence intermittent).

2) Average Thermal Power Output: The average thermal power output for continuous operation (for example) is, from (7) and (3),

$$
\frac{R_{\text {coil }} \int_{T} i_{c}^{2} d t}{|T|} \propto \int_{T} \tau_{c}^{2} d t \propto \int_{T}\left(1-\frac{v_{c}}{v_{\mathrm{nl}, c}}\right)^{2} d t
$$

where the constants of proportionality are the same in both intermittent and continuous modes, so it suffices to compare them with respect to the rightmost quantity. We are interested in the difference in the average thermal power output between continuous and intermittent operation,

$$
D=\int_{A}\left(1-\frac{v_{i}}{v_{\mathrm{nl}, i}}\right)^{2} d t-\int_{T}\left(1-\frac{v_{c}}{v_{\mathrm{nl}, c}}\right)^{2} d t
$$

To compare the thermal effects, we will enforce the condition that the mechanical power output is the same in both modes of operation,

$$
\int_{A} F_{i} v_{i} d t=\int_{T} F_{c} v_{c} d t
$$

We can write the mass's dynamics in the intermittent case as

$$
\int_{T} m \dot{v}_{i} d t=\int_{A} F_{i} d t-\int_{T} m g d t .
$$

Using (11) and (10) in (9), we show in Appendix A that

$$
D=m g|T|\left(\frac{1}{F_{s, i}}-\frac{1}{F_{s, c}}\right) .
$$

3) Comparing $F_{s, i}$ and $F_{s, c}$ : The solution of (4) is increasing in a first-order manner without overshoot, and is asymptotic to the constant $v_{\max }\left(v_{\mathrm{nl}}\right)=a_{1} / a_{2}$.

We argue (cf. Appendix B) that it must be the case that

$$
\frac{d v_{\max }}{d v_{\mathrm{nl}}}>0 \text {. }
$$

Now, one of the following must be true:

1) If $v_{\mathrm{nl}, i}=v_{\mathrm{nl}, c}, \forall$ finite $t \in T, v_{i}(t)<v_{\max }\left(v_{\mathrm{nl}, i}\right)=$ $v_{\max }\left(v_{\mathrm{nl}, c}\right)=v_{c}(t)$.

2) If $v_{\mathrm{nl}, i}<v_{\mathrm{nl}, c}$, using (13) we get $v_{\max }\left(v_{\mathrm{nl}, i}\right)<$ $v_{\max }\left(v_{\mathrm{nl}, c}\right)$. So $\forall$ finite $t \in T, v_{i}(t)<v_{\max }\left(v_{\mathrm{nl}, i}\right)<$ $v_{\max }\left(v_{\mathrm{nl}, c}\right)=v_{c}(t)$.

3) If $v_{\mathrm{nl}, i}>v_{\mathrm{nl}, c}, v_{\max }\left(v_{\mathrm{nl}, i}\right)>v_{\max }\left(v_{\mathrm{nl}, c}\right)$, and it is possible that $\exists t \in T$ s.t. $v_{i}(t)>v_{c}(t)$.

In situations 1 and 2, it is not possible that $\int_{T} v_{i} d t=\int_{T} v_{c} d t$, and intermittent performance is worse than continuous performance. Situation 3 is the only feasible one, i.e., the gear ratio must be lower in intermittent operation for the same mechanical power output in both cases. 
Intuitively, for both systems to have the same average speed, the intermittent system must travel sometimes faster, and sometimes slower than the continuous system. Its $v_{\max }$, and thus $v_{\mathrm{nl}}$, must be larger than that of the continuous system.

This means that

$$
\frac{F_{s, i}}{F_{s, c}}=\frac{v_{\mathrm{nl}, c}}{v_{\mathrm{nl}, i}}<1 .
$$

We can put this in (12) and see that $D>0$. Intermittent operation necessarily produces more thermal energy.

This result represents a fundamental property of motors that cannot be affected by changing the reduction ratio or control scheme. ${ }^{3}$ A number of designers have implemented passive energy storage mechanisms [12], [4] to allow for higher average motor power output and this analytical result further corroborates that such mechanisms are well motivated from a motor-thermal perspective.

\section{Exposing Innate Motor Properties}

Instead of tackling the coupled problems of motor and gearbox selection, the parameterization in III-A allows us to assess and select a motor independent of choice of gear ratio by determining the optimal reduction for each given motor.

1) Optimization Without Thermal Constraints: The motion equations (4) are parameterized by $v_{\mathrm{nl}}$. In order to compare the motors independent of this reduction, we perform a onedimensional optimization over this parameter.

We will ignore the thermal constraints for this subsection only, and focus on relating our performance metric to fundamental motor parameters.

a) Continuous Operation: In continuous operation and in steady state $(F=m g)$, (4) has the simple solution

$$
v_{c}\left(v_{\mathrm{nl}}\right)=v_{\mathrm{nl}}\left(1-\frac{m g v_{\mathrm{nl}}}{4 P}\right),
$$

which is quadratic in $v_{\mathrm{nl}}$ and is maximized when $v_{\mathrm{nl}}=\frac{2 P}{m g}$.

b) Intermittent Operation: For intermittent operation we assume periodic operation (motivated by animals), and fix a "stride length" appropriate in dimension to the pinion gear and mass being lifted by the robot, $x_{r}$. The task is to achieve the maximum average vertical climbing speed in intermittent operation (see Section III-B for definition). Note that the scalar parameter $v_{\text {nl }}$ can be arbitrarily chosen for any motor by varying the reduction $G$, and we will refer interchangeably to "varying $v_{\mathrm{nl}}$ " and "varying $G$ ".

Let us denote the vertical position of the robot, i.e. time integral of the velocity $v$ in (4), as $x=s\left(t, v_{\mathrm{nl}}\right)$, where $s$ : $\mathbb{R}^{+} \times \mathbb{R}^{+} \rightarrow \mathbb{R}$ is obtained by direct integration as

$$
s\left(t, v_{\mathrm{nl}}\right)=\frac{a_{1}}{a_{2}}\left(t+\frac{1}{a_{2}}\left(e^{-a_{2} t}-1\right)\right) .
$$

Note that the release time at which the motor temporarily ceases operation, $t_{r}=\sigma\left(v_{\mathrm{nl}}\right)$ is defined implicitly by $x_{r}=s\left(\sigma\left(v_{\mathrm{nl}}\right), v_{\mathrm{nl}}\right)$. It is easy to show via the implicit function

\footnotetext{
3"Control scheme" here refers to the policy or rule that controls the on/off switching schedule in intermittent operation.
}

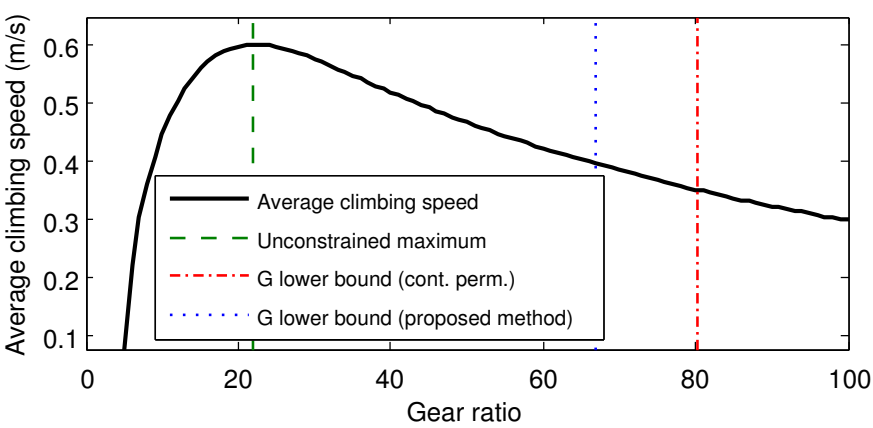

Figure 1. Average climbing speed under periodic intermittent operation as a function of $G$ for Maxon EC45 Flatmotor 251601 [9], showing the (lower) bounds on gear ratio imposed by our proposed method, and following the continuously permissible current specification. The black curve here is that obtained from the mass dynamics in III-C1, and as such over-estimates the climbing performance obtained when restricting current to $i_{\mathrm{cp}}$.

theorem that $\sigma\left(v_{\mathrm{nl}}\right)$ is indeed a function. We numerically find an explicit form of $t_{r}$ by solving $s\left(t, v_{\mathrm{nl}}\right)=x_{r}$. Let us also define $v_{r}\left(v_{\mathrm{nl}}\right):=v\left(t_{r}, v_{\mathrm{nl}}\right)$.

We seek to maximize the average intermittent velocity,

$$
v_{i}\left(v_{\mathrm{nl}}\right)=\frac{x_{r}+\frac{v_{r}^{2}}{2 g}}{t_{r}+\frac{v_{r}}{g}},
$$

but unlike in Section III-C1b, there is no easy calculus solution. This function is not explicit, but we can numerically find an optimal solution (using eg. interior point methods).

2) With Thermal Consequences: In III-C1 we found equations which generate our motor speed-torque trajectories, parameterized by gear ratio $G$. By using a thermal model for our motor and leveraging our freedom in $G$, we can dynamically generate trajectories of operation-ensuring thermal constraints are satisfied - based on a more abstract task specification, using our thermal model in the analysis itself.

We use the thermal model formulation of III-A3, where $F\left(t, v_{\mathrm{nl}}\right)=m \dot{v}\left(t, v_{\mathrm{nl}}\right)$ in (5) is given by the equations of motion in III-A2.

For continuous operation in steady state, (5) and (7) give

$$
\bar{\iota}_{c}\left(t, v_{\mathrm{nl}}\right)=\frac{R_{\mathrm{coil}} v_{\mathrm{nl}}^{2}(m g)^{2}}{K_{T}^{2} \omega_{\mathrm{nl}}^{2}}
$$

while for intermittent operation with stride length $x_{r}$ as defined in Section III-C1b, we get

$$
\bar{\iota}_{i}=\frac{R_{\mathrm{coil}} v_{\mathrm{nl}}^{2} m^{2}}{K_{T}^{2} \omega_{\mathrm{nl}}^{2}\left(t_{r}+v_{r} / g\right)} \int_{0}^{t_{r}}\left(g+a_{1} e^{-a_{2} t}\right)^{2} d t .
$$

Using (16) or (17) in (8) we can compute the temperature rise and limit our optimization in Section III-C to gear ratios that keep the core temperature under acceptable limits. ${ }^{4}$

\footnotetext{
${ }^{4}$ Note that, intuitively, an arbitrarily large gear ratio will allow any force to be generated with very small motor currents. Thus, there always exists a "feasible" gear ratio.
} 


\section{Simulation Results}

In order to test our motor sizing method for use in practice, we performed numerical trials on all of the motors in [9]. We envision that these simulations will not only enable robot designers to pick out exactly which commercially available motor to use for their robot, but also give feedback about the type of gearbox needed as well as the performance to expect.

\section{A. Comparing Motor-Sizing Methods}

A central contribution of this paper is the use of a dynamical systems task model that yields a closed form family of trajectories over all possible intitial conditions, and, hence, enables motor and gearbox selection over the entire family. Absent this analytically determined design insight, the recourse would be to approximate the "typical" robot speed-torque requirements across a range of "reasonable" example trajectories and iterate through motor/gearbox combinations. Here we assume that the ideas of III-C1 are being used, and compare the effect of including the thermal model of III-C2 in the design process.

We use our simulation to find the best possible vertical climbing (average) speed that can be obtained by picking the gear ratio (a) without thermal considerations, (b) using the continuously permissible current motor specification, and (c) with a dynamic thermal model (cf. III-C2).

For a specific motor, Fig. 1 demonstrates the nature of the objective function (15) as a function of $G$, as well as the lower bounds imposed by our thermal constraint (cf. III-C2), as well as that required to keep motor current under $i_{\mathrm{cp}}$. Note that the latter is more restrictive, while disregarding the constraint entirely gives the best performance (albeit, unacceptably damaging to the motor).

See Fig. 2 for a scatter plot of performance exhibited by the motors in these cases. Note that we have limited the motors displayed to those that can perform the task with a reduction $G \leq 100$. This restricts our results to those motors for which the task parameters (mass and stride length) are roughly appropriate ${ }^{5}$ to the size of the motor.

For our simulations of intermittent operation, we chose the periodic leaping motion defined by fixing a "stride length" as described in Section III-C1b.

We compare our results with those obtained by limiting motor coil current to be under the "continuously permissible current" motor specification, $i_{\mathrm{cp}}$. In continuous operation, motor force $F=m g$, and we need to ensure

$$
i_{\mathrm{m}}=\frac{v_{\mathrm{nl}} m g}{K_{T} \omega_{\mathrm{nl}}} \leq i_{\mathrm{cp}} \Longrightarrow v_{\mathrm{nl}} \leq \frac{i_{\mathrm{cp}} K_{T} \omega_{\mathrm{nl}}}{m g}
$$

From the result in Fig. 2-A we see that in continuous operation, not considering the thermal effects in the design process [13] allows us to pick a gear ratio which promises very good performance, but would result in thermal damage to the

\footnotetext{
${ }^{5}$ Situations like (for eg.) a nominal $1 \mathrm{~W}$ motor attempting to cause a $50 \mathrm{~kg}$ mass to leap result in extreme gear ratios and/or performance figures that are difficult to represent (owing to plot axis scaling) and phyically impractical.
}
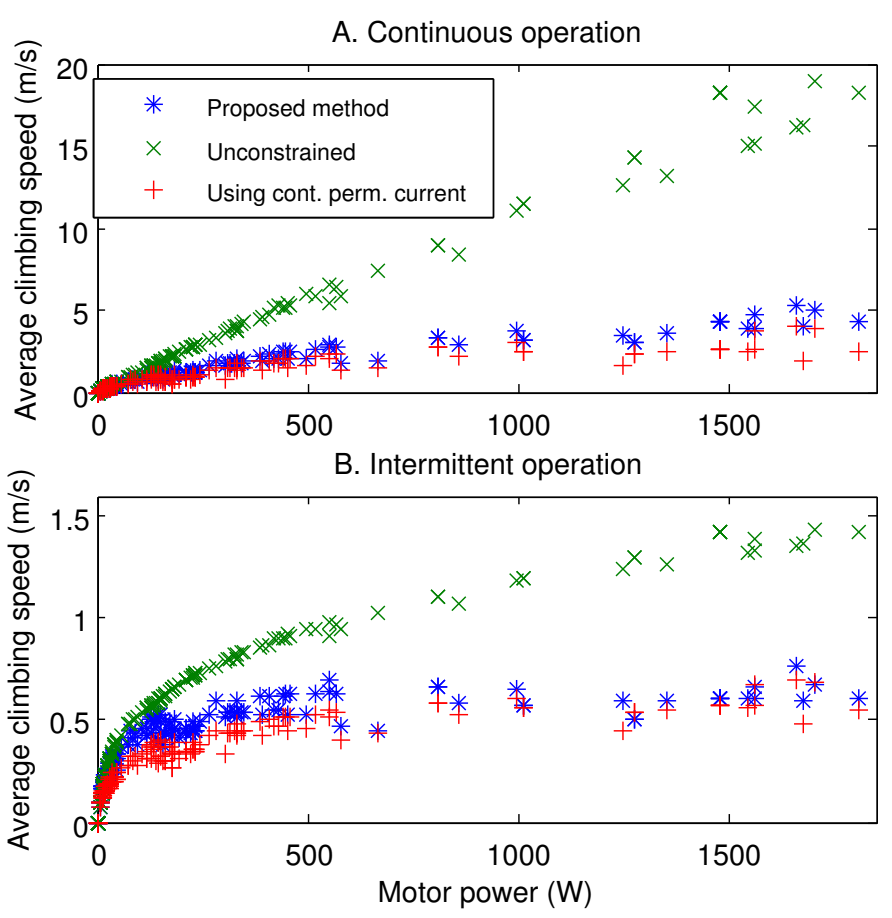

Figure 2. A comparison of the best vertical climbing performance possible picking the gear reduction with the method proposed in this paper, and conventional methods (no thermal constraint, and using continuously permissible current), in continuous (A) and intermittent (B) operation.

motor. ${ }^{6}$ More interestingly, our method allows us to achieve higher performance than is reachable by restricting motor coil current to values below the continuously permissible current specification of the motor [12], [7].

For intermittent operation with a fixed voltage, note that the motor must start from its stall torque (irrespective of the reduction ratio). This means that it is impossible to ensure that the coil current is under the continuously permissible current spec. We chose a slightly less naïve control strategy that uses varying voltage to keep the motor current under $i_{\mathrm{cp}}$. Once the trajectory reaches the highest speed-torque curve (voltage is equal to the maximum supply voltage), we use the same equations as in Section III-Clb.

From the performance results displayed in Fig. 2, we note that gearbox selection using a dynamic thermal model (cf. III-C2) results in average vertical speed improvement (mean \pm std. dev.) of $21.6 \% \pm 19.0 \%$ (continuous) and $27.7 \% \pm 12.3 \%$ (intermittent) when compared to restricting coil current to $i_{\mathrm{cp}}$. The maximum improvement seen in intermittent operation was $57.6 \%$. Thus, by explicitly considering thermal behavior instead of simply restricting current to continuously permissible levels, we achieved notably better performance and correspondingly, higher average motor power outputs.

The additional performance improvements from our optimization in the intermittent case are attributable to taking

\footnotetext{
${ }^{6}$ From the analysis of Section III-C2, the blue $(*)$ points correspond to the fastest average speed that can be obtained without overheating the motor core, so all the green $(x)$ points that lie above blue $(*)$ points indicate a gearbox selection that will result in thermal damage.
} 

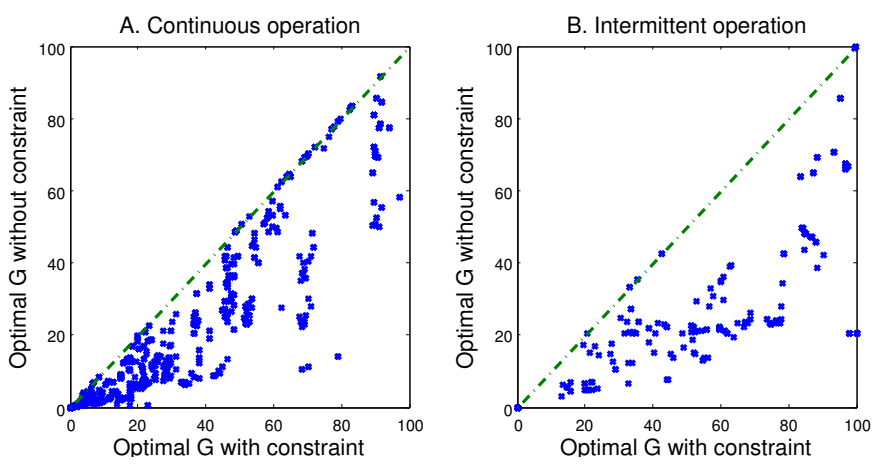

Figure 3. Optimal gear ratios for a subset of the motors, showing how adding the thermal constraints requires a higher reduction. Note that there are fewer motors shown in B as several motors from [9] required a much higher gear ratio due to the intermittence penalty described in Section III-B.

advantage of the short "refractory periods." While the torquerestricted climber is limited to its continuously permissible current at all times, our thermal model permits us to select a gearbox with the expectation of exceeding the continuously acceptable current and then "resting." These benefits would undoubtedly be amplified were we to consider thermal constraints over a task with a finite completion time (eg. a specified number of strides), as the thermally optimal behavior would expend a "thermal budget" over the duration of the task, an effect not seen here due to the infinite task timeline.

\section{B. Thermal Considerations and Gearbox Selection}

In Fig. 3, we see the effect our thermal constraint has on the selection of $v_{\mathrm{nl}}$, and thus $G$. Adding thermal considerations necessitates choosing a higher $G$ in order to keep motor torque (and current) lower for the same output torque. Thus, all data points are to the right of the $45^{\circ}$-line in both parts of Fig. 3. Intermittent operation carries an inherent thermal "penalty" (see Section III-B), resulting in a further rightward shift of the data points from Fig. 3-A to Fig. 3-B.

We envision that information similar to what is presented above will be useful to the designer for (a) the identification of motors which can fulfil a given task without needing to recourse to a physically unreasonable gear ratio, and (b) gearbox selection for a specific motor.

\section{CONCLUSiON}

We have outlined a principled approach (if not an algorithm) to tackle the coupled problems of motor and gearbox selection from both mechanical and thermal dynamic perspectives. In so doing, we introduce a novel concept of task specification and proceed by leveraging optimization, dynamic simulation, and analysis tools. We achieve an analytical result, relevant to any task reasonably well characterized by our simple vertical leaping dynamics, demonstrating the cost of intermittent motor operation. Our result further justifies roboticists' previous work on the introduction of passive-elastic energy storage to enable more continuous actuator power delivery [4], [14], and gives strong motivation to promote-through mechanical design - an approximately constant motor speed.
To develop and demonstrate our method, we used as an example a 1-DOF vertical climbing robot (with trivial dynamics). Even though this task appears restrictive, it generates motor trajectories across a broad range of regimes in the (fixedvoltage) speed-torque operational plane. Even for applications with strikingly different requirements, a computation akin to III-C can be used to determine the set of motor characteristics that play a similarly crucial role in affecting the execution quality of that task, as well as enable the development of motor-gearbox selection aids akin to Figs. 2-3.

\section{ACKNOWLEDGEMENTS}

This work was funded in part by the National Science Foundation under FIBR award 0425878 and in part by the US Army Research Lab Robotics Collaborative Technology Alliance.

The authors would like the thank Albert Kwon for parsing the Maxon motor catalog [9], which was used extensively in generating the numerical results.

\section{REFERENCES}

[1] J. Clark, G. Lynch, and D. Koditschek, "A bio-inspired dynamical vertical climbing robot," in preparation.

[2] K. C. Galloway, G. C. Haynes, B. D. Ilhan, A. M. Johnson, R. Knopf, G. Lynch, B. Plotnick, M. White, and D. E. Koditschek, "X-rhex: A highly mobile hexapedal robot for sensorimotor tasks," University of Pennsylvania, Tech. Rep., 2010.

[3] U. Kafader, The Selection of High-Precision Microdrives. Maxon Academy, 2006.

[4] J. Clark, D. Goldman, P. Lin, G. Lynch, T. Chen, H. Komsuoglu, R. Full, and D. Koditschek, "Design of a bio-inspired dynamical vertical climbing robot," in Proceedings of Robotics: Science and Systems, Atlanta, GA, USA, June 2007.

[5] P. Gregorio, M. Ahmadi, and M. Buehler, "Design, control, and energetics of an electrically actuated legged robot," Systems, Man, and Cybernetics, Part B: Cybernetics, IEEE Transactions on, vol. 27, no. 4, pp. $626-634$, aug. 1997.

[6] I. Poulakakis, J. A. Smith, and M. Buehler, "Experimentally validated bounding models for the scout ii quadrupedal robot," in ICRA, 2004, pp. 2595-2600.

[7] V. Potkonjak, "Thermal analysis and dynamic capabilities of DC motors in industrial robotic systems," Robotics and Computer-Integrated Manufacturing, vol. 5, no. 2-3, pp. 137 - 143, 1989.

[8] S. E. Zocholl, "Optimizing Motor Thermal Models," in Petroleum and Chemical Industry Conference, 2006. PCIC'06. Record of Conference Papers-IEEE Industry Applications Society 53rd Annual. IEEE, 2007, pp. 1-9.

[9] Maxon Motors, "Maxon Catalog, Key Information," pp. 36-43, 20092010.

[10] D. DeWitt and F. Incropera, Fundamentals of heat and mass transfer. Wiley New York, 1996.

[11] M. H. Dickinson, C. T. Farley, R. J. Full, M. A. n. Koehl, R. Kram, and S. Lehman, "How animals move: An integrative view," Science, vol. 288, no. 5463, pp. 100-106, 2000.

[12] J. E. Clark and D. E. Koditschek, "A spring assisted one degree of freedom climbing model," Lecture Notes on Control and Information Sciences, pp. 43-64, 2006.

[13] D. Wollherr, M. Hardt, M. Buss, and O. von Stryk, "Actuator selection and hardware realization of a small and fast-moving autonomous humanoid robot," in Proc. 2002 IEEE/RSJ Int. Conf. on Intelligent Robots and Systems (IROS). Citeseer, 2002.

[14] M. H. Raibert, "Dynamic stability and resonance in a one-legged hopping machine," in 4th Symp. Theory and Practice of Robots and Manipulators, 1981. 


\section{APPENDIX A}

Proof of Intermittence Penalty

Equal average mechanical power output implies (from 10)

$$
\int_{A} F_{i} v_{i} d t=\int_{T} F_{c} v_{c} d t
$$

For steady state rolling, $F_{c}=m g$. Suppose the height gained in continual operation is $h$. Then the right hand side of (10) is equal to $\mathrm{mgh}$.

Combining these with the motor kinematics (3), we get

$$
F_{s, i} \int_{A} v_{i}\left(1-\frac{v_{i}}{v_{\mathrm{nl}, i}}\right) d t=m g h,
$$

or

$$
\int_{A}\left(-\frac{v_{i}}{v_{\mathrm{nl}, i}}+\frac{v_{i}^{2}}{v_{\mathrm{nl}, i}^{2}}\right) d t=-\frac{m g h}{4 P},
$$

remembering from (1) that $F_{s, i} v_{\mathrm{nl}, i}=4 P$ where $P$ is the constant peak motor power (irrespective of gearbox).

The discrepancy in average thermal power output is,

$$
\begin{aligned}
D & =\int_{A}\left(1-\frac{v_{i}}{v_{\mathrm{nl}, i}}\right)^{2} d t-\int_{T}\left(1-\frac{v_{c}}{v_{\mathrm{nl}, c}}\right)^{2} d t \\
& =\int_{A}\left(1-\frac{2 v_{i}}{v_{\mathrm{nl}, i}}+\frac{v_{i}^{2}}{v_{\mathrm{nl}, i}^{2}}\right) d t-\frac{m g}{F_{s, c}}\left(1-\frac{v_{c}}{v_{\mathrm{nl}, c}}\right) \int_{T} d t .
\end{aligned}
$$

In the mass dynamics from (11),

$$
\begin{aligned}
\int_{T} m \dot{v}_{i} d t & =\int_{A} F_{i} d t-\int_{T} m g d t \\
\Longrightarrow \int_{A} F_{i} d t & =m g|T| .
\end{aligned}
$$

We assumed here that the net change in momentum of the mass over the interval is zero. This will obviously happen if the intermittent motion is periodic and the time interval covers a whole number of periods.

Using this in (9),

$$
\begin{aligned}
D & =\int_{A}\left(1-\frac{v_{i}}{v_{\mathrm{nl}, i}}\right) d t-\frac{m g h}{4 P}-\left(\frac{m g|T|}{F_{s, c}}-\frac{m g\left(|T| v_{c}\right)}{F_{s, c} v_{\mathrm{nl}, c}}\right) \\
& =\int_{A} \frac{F_{i}}{F_{s, i}} d t-\frac{m g|T|}{F_{s, c}},
\end{aligned}
$$

where we used the notation $|T|=\int_{T} d t$, noted that $|T| v_{c}=h$, $F_{s, c} v_{\mathrm{nl}, c}=4 P$, and used (19) above. Now using (21), we get (12).

\section{APPENDIX B}

\section{ARGUMENT THAT $v_{\mathrm{MAX}}$ INCREASES WITH $v_{\mathrm{NL}}$}

The reasoning is that, for continual operation, it must be true that we were thermally limited. ${ }^{7}$ Recall from (5) that the RMS thermal constraint gives an upper bound on $v_{\mathrm{nl}}$ If (13)

\footnotetext{
${ }^{7}$ If continual operation were not thermally limited, we could set our reduction ratio so as to harvest the peak motor power for the whole interval $T$. In this case, it would be impossible for intermittent operation to match it.
}

were not true, in the continual operation case we could get better performance by reducing $v_{\mathrm{nl}}$, which is a contradiction because we assume we are already operating at the optimal $v_{\mathrm{nl}}$. 